\title{
The Prognostic Relevance of Computed Tomography-assessed Skeletal Muscle Index and Skeletal Muscle Radiation Attenuation in Patients With Gynecological Cancer
}

\author{
ANGIOLO GADDUCCI and STEFANIA COSIO \\ Department of Clinical and Experimental Medicine, \\ Division of Gynecology and Obstetrics, University of Pisa, Pisa, Italy
}

\begin{abstract}
The evaluation of the whole skeletal muscle area at the level of the third lumbar vertebra on computed tomography (CT) scans has often detected loss of skeletal muscle mass, defined as sarcopenia, and reduced skeletal muscle radiation attenuation (SMRA) in patients with different malignancies. Baseline sarcopenia has been detected in $33.3 \%-51.8 \%$ of patients with advanced cervical cancer, $33.6 \%-50 \%$ of those with endometrial cancer, and 11\%-64\% of those with advanced ovarian cancer. We reviewed the literature data on the clinical relevance of CT-assessed skeletal muscle status in gynecological malignancies. Overall, baseline skeletal muscle index and SMRA have an uncertain prognostic relevance, whereas their changes during treatment usually correlate with progression-free survival and overall survival. Multicenter clinical trials are strongly warranted to assess the effects of pharmacological agents and physical exercise in the management of skeletal muscle damage in patients with gynecological cancer.
\end{abstract}

Cancer cachexia is a wasting syndrome characterized by loss of skeletal muscle mass and functional strength defined as sarcopenia with or without loss of fat mass, associated with anorexia, inflammation, insulin resistance, and decreased quality of life $(1,2)$. Mitochondria, which produce adenosine

This article is freely accessible online.

Correspondence to: Angiolo Gadducci, MD, Department of Clinical and Experimental Medicine, Division of Gynecology and Obstetrics, University of Pisa, Via Roma 56, Pisa, 56127, Italy. Tel: +39 50992609, e-mail: a.gadducci@med.unipi.it

Key Words: Sarcopenia, skeletal muscle index, skeletal muscle radiation attenuation, cervical cancer, endometrial cancer, ovarian cancer, review. triphosphate (ATP) through oxidative phosphorylation and beta-oxidation, have a pivotal role in the pathogenesis of cachexia $(3,4)$. Elevated Interleukin (IL)-6, Tumor Necrosis Factor (TNF)- $\alpha$ and Transforming Growth Factor (TGF)- $\beta$ levels affect mitochondrial homeostasis. Dysfunctional mitochondria release reactive oxygen species and decrease ATP production, thus leading to enhanced protein catabolism and decreased muscle mass.

In an experimental murine model of human ovarian cancer, cachexia was associated with high tumor-derived IL6 levels in plasma and ascites as well as with elevated phospho- signal transducer and activator of transcription proteins $(\mathrm{STAT})_{3}$, decreased phospho-AKT and increased protein ubiquitination and expression of ubiquitin ligases in skeletal muscles of tumor hosts (5). Therefore IL-6-induced $\mathrm{STAT}_{3}$ activation appears to be involved in muscle wasting.

With the introduction of methods of in vivo body composition evaluation such as magnetic resonance imaging (MRI) and computed tomography (CT), the measurement of skeletal muscle mass with cross-sectional imaging has become a very common tool of sarcopenia assessment, although there is a wide heterogeneity in both the parameters taken into consideration and the diagnostic criteria used by different authors (6-18). The assessment of psoas muscle area-only is easy and quick, but it is not representative of the total body skeletal muscle (19). Moreover, degenerative diseases of the lumbar spine can cause local atrophy of the trunk muscles and psoas muscle not specifically related to cancer-induced sarcopenia. Conversely, the evaluation of whole skeletal muscle area at the level of the third lumbar vertebra is a more reliable and widely validated method for the assessment of the total body skeletal muscle (20-22), especially in cancer patients $(6,23-28)$. The cross-sectional area of skeletal muscles normalized for the patient height to calculate the skeletal muscle index (SMI) (23). Sarcopenia is usually defined as an SMI lower than the selected cut-off value. 
Table I. Baseline skeletal muscle assessment by CT scan in locally advanced cervical carcinoma.

\begin{tabular}{lccccc}
\hline Authors & Pts & FIGO stage & Landmark & SM assessment & Percentage \\
\hline Lee et al. (23) & $245^{\mathrm{a}}$ & Ib -IV or pelvic N+ & L3 & $\begin{array}{c}\text { SMI } \leq 41 \mathrm{~cm}^{2} / \mathrm{m}^{2} \\
\text { SMRA }<41 \text { HU if BMI }<25.0 \mathrm{~kg} / \mathrm{m}^{2} \\
\text { or }<33 \text { HU if BMI } \geq 25.0 \mathrm{~kg} / \mathrm{m}^{2}\end{array}$ & $\begin{array}{c}51.8 \% \\
62.9 \%\end{array}$ \\
Matsuoka et al. (11) & $236^{\mathrm{b}}$ & Ib1-IVa & L3 & $\begin{array}{l}\text { Median SMI } 36.5 \mathrm{~cm}^{2} / \mathrm{mm}^{2} \\
\text { Median PMI } 3.94 \mathrm{~cm}^{2} / \mathrm{mm}^{2}\end{array}$ \\
Sanchez et al. (32) & $55^{\mathrm{a}}$ & II-III & L3 & SM $<38.5 \mathrm{~cm}^{2} / \mathrm{m}^{2}$ & $33.3 \%$ \\
Kiyotoki et al. (28) & $60^{\mathrm{a}}$ & Ib -IVa & L3 & Median SM $90.29 \mathrm{~cm}^{2}$ & Median PM $10.07 \mathrm{~cm}^{2}$ \\
\hline
\end{tabular}

a Concurrent chemoradiotherapy; bConcurrent chemoradiotherapy or radiotherapy. CT: Computed tomography; pts: patients; SM: skeletal muscle; N: lymph nodes; L3: third lumbar vertebra; SMI: skeletal muscle index; SMRA: skeletal muscle radiation attenuation; HU: Hounsfield Units; BMI: body mass index; PMI: psoas muscle index.

Radiation attenuation, which is tissue-specific, ranges from -190 to -30 Hounsfield Units (HU) for adipose tissue and from -29 to $+150 \mathrm{HU}$ for muscle, which allows to discriminate fat from muscle and to quantify fatty muscle infiltration (29). Skeletal muscle contains lipid droplets within the cytoplasm of myocytes as well as intermuscular adipocytes. Muscles of cancer patients often show an increase of intramyocellular lipid droplets, which are more abundant in patients with progressive cancer-related weight loss compared to weight-stable individuals (30). A low skeletal muscle radiation attenuation (SMRA) associated with lipid accumulation has been often observed in CT scans of cancer patients $(29,31)$.

Sarcopenia is an index of frailty associated with longer hospital stay, higher risk of surgical complications, increase chemotherapy toxicity and unfavorable prognosis in patients with different malignancies including breast cancer $(6,9)$, pancreatic cancer (10), colon cancer (13), cholangiocarcinoma (14) and oropharyngeal squamous cell carcinoma (15). For instance, Shachar et al. (9), who assessed 40 metastatic breast cancer women receiving first-line taxane-based chemotherapy, found grade 3-4 toxicity in $57 \%$ and $18 \%$, respectively, of sarcopenic and non-sarcopenic patients $(p=0.02)$. Among 55 women with metastatic breast cancer resistant to anthracycline and/or taxane, Prado et al. (6) reported capecitabine-related toxicity in $50 \%$ of sarcopenic versus $20 \%$ of non-sarcopenic patients $(p=0.03)$. The impact of sarcopenia in patients with malignancies of the female genital tract has yet to be clearly elucidated $(12,16,17,19,23,26,28)$.

Since CT is commonly used for staging, assessment of response to treatment and surveillance of gynecological cancers, this imaging technique can offer useful information on the prognostic relevance of baseline and post-treatment SMI and SMRA in patients with these malignancies. The aim of the present article is to review the literature data on the clinical relevance of CT-assessed skeletal muscle status in locally advanced cervical cancer, endometrial cancer and advanced ovarian cancer.

\section{Cervical Cancer}

Baseline sarcopenia has been detected in 33.3\%-51.8\% of patients with locally advanced cervical cancer $(11,23,28$, 32) (Table I). This variable has no impact on the clinical outcome, whereas SMI decrease during definitive radiotherapy or concurrent chemo-radiotherapy significantly correlated with poorer prognosis (Tables II and III).

In the study of Lee et al. (23) baseline sarcopenia was defined as an SMI of $\leq 41.0 \mathrm{~cm}^{2} / \mathrm{m}^{2}$ and a low SMRA was defined as a mean attenuation of $<41 \mathrm{HU}$ in patients with a body mass index [BMI] of $<25.0 \mathrm{~kg} / \mathrm{m}^{2}$ or $<33 \mathrm{HU}$ in patients with a BMI of $\geq 25.0 \mathrm{~kg} / \mathrm{m}^{2}$ in agreement with Martin and coworkers (24). During concurrent chemoradiotherapy the patients lost an average of $0.6 \%$ of SMI 150 days, and the incidence of SMI loss was higher in patients with adenocarcinoma than in those with squamous cell carcinoma $(43.3 \%$ versus $17.2 \%, p=0.003)$. Most studies have reported that adenocarcinoma has a more aggressive biological behavior and a poorer clinical outcome compared with squamous cell carcinoma (33), and the higher skeletal loss may be a mechanism by which adenocarcinoma can detrimentally impact on patient prognosis (23). Baseline low SMI and low SMRA were not associated with the clinical outcome. An SMI loss during treatment $>10.0 \% / 150$ days had a detrimental impact on both overall survival (OS) [hazard ratio $(\mathrm{HR})=6.02 ; p<0.001$ ] and cancer specific survival $(\mathrm{CSS})(\mathrm{HR}=3.49 ; p=0.006)$ at multivariate analysis, whereas SMRA change was not an independent predictor for either progression-free survival (PFS) or OS. 
Table II. Prognostic relevance of skeletal muscle assessment by CT scan in locally advanced cervical carcinoma: Baseline assessment.

\begin{tabular}{|c|c|c|c|c|c|c|c|c|}
\hline \multirow[t]{2}{*}{ Author } & \multicolumn{3}{|c|}{ Clinical outcome: PFS } & \multicolumn{3}{|c|}{ Clinical outcome: OS } & \multicolumn{2}{|c|}{ Clinical outcome: CSS } \\
\hline & & & & & ar survival & $p$-Value & 5-year survival & $p$-Value \\
\hline \multicolumn{9}{|l|}{ Lee et al. (23) } \\
\hline Low vs. high SMI & & & & \multirow{2}{*}{\multicolumn{2}{|c|}{$\begin{array}{c}82.6 \% \text { vs. } 83 \% \\
89.9 \% \text { vs. } 86.1 \%\end{array}$}} & 0.68 & $87.9 \%$ vs. $86.6 \%$ & $0.84 *$ \\
\hline \multirow[t]{2}{*}{ Low vs. high SMRA } & & & & & & 0.26 & $87.4 \%$ vs. 87.11 & $0.84 *$ \\
\hline & HR & $95 \% \mathrm{CI}$ & $p$-Value & $\mathrm{HR}$ & $95 \% \mathrm{CI}$ & $p$-Value & & \\
\hline \multicolumn{9}{|l|}{ Matsuoda et al. (11) } \\
\hline Low vs. high SMI & 1.143 & $0.738-1.773$ & $0.549 *$ & 1.126 & $0.697-1.818$ & $0.628^{*}$ & & \\
\hline Low vs. high PMI & 1.176 & $0.758-1.823$ & $0.469 *$ & 1.118 & $0.692-1.805$ & $0.648^{*}$ & & \\
\hline \multicolumn{9}{|l|}{ Kiyotoki et al. (28) } \\
\hline Low $v s$. high SM & & & $0.738 *$ & & & $0.376^{*}$ & & \\
\hline Low $v s$. high PM & & & $0.958 *$ & & & $0.515^{*}$ & & \\
\hline
\end{tabular}

*Univariate analysis; **multivariate analysis. CT: Computed tomography; SMI: skeletal muscle index; OS: overall survival; CSS: cancer specific survival; PFS: progression-free survival; HR: hazard ratio; 95\%CI: 95\% confidence interval; PMI: psoas muscle index; SM: skeletal muscle.

Table III. Prognostic relevance of skeletal muscle assessment by CT scan in locally advanced cervical carcinoma: Changes during treatment.

\begin{tabular}{|c|c|c|c|c|c|c|c|c|c|}
\hline \multirow[t]{2}{*}{ Author } & \multicolumn{3}{|c|}{ Clinical outcome PFS } & \multicolumn{3}{|c|}{ Clinical outcome OS } & \multicolumn{3}{|c|}{ Clinical outcome CSS } \\
\hline & & & & \multicolumn{2}{|c|}{ 5-year survival } & $p$-Value & \multicolumn{2}{|c|}{ 5-year survival } & $p$-Value \\
\hline \multirow{3}{*}{$\begin{array}{l}\text { Lee } \text { et al. }(23) \\
\text { SMI loss }>10 \% \text { vs. stable } \\
\text { SMI } v \text { s. SMI gain }\end{array}$} & & & & \multirow{2}{*}{\multicolumn{2}{|c|}{$\begin{array}{c}45.2 \% \text { vs. } 91.2 \% \\
\text { vs. } 95.6 \%\end{array}$}} & \multirow{3}{*}{$<0.001 *$} & \multirow{2}{*}{\multicolumn{2}{|c|}{$\begin{array}{c}59.8 \% \text { vs. } 92.6 \% \\
\text { vs. } 95.6 \%\end{array}$}} & \multirow{3}{*}{$<0.001 *$} \\
\hline & & & & & & & & & \\
\hline & & & & HR & $95 \% \mathrm{CI}$ & & HR & $95 \% \mathrm{CI}$ & \\
\hline \multirow{4}{*}{$\begin{array}{l}\text { SMRA change } \\
\text { (per 1HU increase) }\end{array}$} & & & & 6.02 & $3.04-11.93$ & $<0.001 * *$ & 3.49 & $1.44-8.42$ & $0.006 * *$ \\
\hline & & & & 0.80 & $0.74-0.88$ & $<0.001 *$ & 0.85 & $0.76-0.94$ & $0.001 *$ \\
\hline & & & & 0.95 & $0.87-1.03$ & $0.19 *$ & 0.92 & $0.82-1.05$ & $0.21 * *$ \\
\hline & HR & $95 \% \mathrm{CI}$ & $p$-Value & HR & $95 \% \mathrm{CI}$ & $p$-Value & & & \\
\hline \multicolumn{10}{|l|}{ Sanchez et al. (32) } \\
\hline SMI loss $>10 \%$ & $2.957^{\mathrm{a}}$ & - & 0.006 & $2.572^{\mathrm{b}}$ & & 0.06 & & & \\
\hline \multicolumn{10}{|l|}{ Kiyotoki et al. (28) } \\
\hline \multirow[t]{2}{*}{ SM loss $>15 \%$} & 4.714 & $1.860-11.947$ & $<0.001^{*}$ & 6.035 & $2.182-16.69$ & $0.001 *$ & & & \\
\hline & 1.619 & $0.527-4.971$ & $0.4^{* *}$ & 2.892 & $0.744-11.240$ & $0.125 * *$ & & & \\
\hline \multirow[t]{2}{*}{ PM loss $>15 \%$} & 6.638 & $2.651-16.620$ & $0.001 *$ & 12.571 & $4.403-35.893$ & $<0.001 *$ & & & \\
\hline & 6.001 & 1.90818 .871 & $0.002 * *$ & 8.515 & $2.159-33.585$ & $0.002 * *$ & & & \\
\hline
\end{tabular}

*Univariate analysis; **multivariate analysis. CT: Computed tomography; SMI: skeletal muscle index; OS: overall survival; CSS: cancer specific survival; PFS: progression-free survival; HR: hazard ratio; 95\%CI: 95\% confidence interval; PMI: psoas muscle index; SM: skeletal muscle. ${ }^{\text {aHigher }}$ tumor recurrence; ${ }^{\mathrm{b}}$ Trend to reduced OS.

A retrospective Japanese study found that pretreatment SMI and psoas muscle index [PMI] (i.e. psoas muscle normalized for the patient height) significantly correlated with parametrial status ( $p=0.034$ and $p=0.002$ ) but not with PFS and OS (11). An observational prospective Mexican study revealed that at diagnosis no patients were malnourished although $33.3 \%$ presented sarcopenia, whereas at the end of treatment $69 \%$ were malnourished and $58 \%$ were sarcopenic (32). The patients who lost $\geq 10 \%$ of SMI experienced a significantly higher recurrence rate 
Table IV. Skeletal muscle assessment by CT scan and its prognostic relevance in endometrial carcinoma: Baseline assessment.

\begin{tabular}{|c|c|c|c|c|c|}
\hline Authors & Pts & FIGO stage & Landmark & SM assessment & Percentage \\
\hline Kukori et al. (8) & $122^{\mathrm{a}}$ & I-IV & L3 & $\mathrm{SMA} \leq 4.33 \mathrm{~cm}^{2}$ & $50.0 \%$ \\
\hline Rodriguez et al. (34) & $208^{b}$ & I-IV & L3 & $\begin{array}{c}\text { Median SMI } 42.45 \mathrm{~cm}^{2} / \mathrm{m}^{2} \\
\text { Median SMRA } 30 \mathrm{HU}\end{array}$ & \\
\hline Lee et al. (35) & $131^{\mathrm{c}}$ & III & L3 & $\begin{array}{c}\mathrm{SMI}<39.3 \mathrm{~cm}^{2} / \mathrm{m}^{2} \\
\text { SMRA }<33 \mathrm{HU}\end{array}$ & $33.6 \%$ \\
\hline Ganju et al. (36) & $64^{\mathrm{d}}$ & Ib-IVa & L3 & $\begin{array}{c}\text { SMI }<41 \mathrm{~cm}^{2} / \mathrm{m}^{2}: \\
\text { SMRA }<41 \mathrm{HU} \text { if BMI }<25 \mathrm{~kg} / \mathrm{m}^{2} \text { and } \\
\text { SMRA }<33 \mathrm{HU} \text { if } \mathrm{BMI}>25 \mathrm{~kg} / \mathrm{m}^{2}\end{array}$ & $\begin{array}{l}44 \% \\
80 \%\end{array}$ \\
\hline
\end{tabular}

asurgery, 122; adjuvant treatment, radiotherapy, 18; chemotherapy, 27; chemotherapy/radiotherapy, 19; bSurgery, 111; surgery plus chemotherapy, 66; palliative treatment, 31; 'S Surgery plus chemotherapy and radiotherapy; dSurgery plus radiotherapy with (n. 25) or without chemotherapy.

Table V. Skeletal muscle assessment by CT scan and its prognostic relevance in endometrial carcinoma: Prognostic relevance.

\begin{tabular}{|c|c|c|c|c|c|}
\hline \multicolumn{6}{|l|}{ Baseline assessment } \\
\hline \multirow[t]{2}{*}{ Author } & Clinical outcome PFS & \multirow[b]{2}{*}{$p$-Value } & \multicolumn{2}{|c|}{ Clinical outcome OS } & \multirow[b]{2}{*}{$p$-Value } \\
\hline & $95 \% \mathrm{CI}$ & & HR & $95 \% \mathrm{CI}$ & \\
\hline \multicolumn{6}{|l|}{ Kukori et al. (8) } \\
\hline \multirow[t]{2}{*}{ Low vs. high SMA } & Median 23.5 vs. 32.1 months & $0.046^{*}$ & \multicolumn{2}{|c|}{ median 29.4 vs. 33.9 months } & $0.587 *$ \\
\hline & $1.42-11.3$ & $\mathrm{NA}^{* *}$ & 1.98 & $0.81-4.86$ & $\mathrm{NA}^{* *}$ \\
\hline \multicolumn{6}{|l|}{ Rodriguez et al. (34) } \\
\hline High SMI + high SMRA & & & \multicolumn{2}{|c|}{1 (reference) } & $0.004 * *$ \\
\hline Low SMI + high SMRA & & & 2.10 & $0.48-9.16$ & \\
\hline High SMI + low SMRA & & & 2.20 & $0.67-7.16$ & \\
\hline Low SMI + low SMRA & & & 5.31 & $1.71-16.51$ & \\
\hline \multicolumn{6}{|l|}{ Lee et al. (35) } \\
\hline Low $v s$. high SMRA & $70.5 \%$ vs. $80.7 \%$ & $0.24 *$ & \multicolumn{2}{|c|}{$76.7 \%$ vs. $81.3 \%$} & $0.47 *$ \\
\hline \multicolumn{6}{|l|}{ Ganju et al. (36) } \\
\hline Low $v s$. high SMI & & & 2.42 & $0.87-6.72$ & $0.09 *$ \\
\hline Low $v s$. high SMRA & & & 3.52 & $0.81-15.3$ & $0.09 *$ \\
\hline \multirow[t]{2}{*}{ Low SMI + low SMRA } & & & 4.25 & $1.53-11.79$ & $<0.01 *$ \\
\hline & & & 3.02 & $1.04-8.74$ & $0.04 * *$ \\
\hline \multicolumn{6}{|l|}{ Changes after treatment } \\
\hline \multirow[t]{2}{*}{ Author } & Clinical outcome PFS & & \multicolumn{2}{|c|}{ Clinical outcome OS } & \\
\hline & $95 \% \mathrm{CI}$ & $p$-Value & HR & $95 \% \mathrm{CI}$ & $p$-Value \\
\hline \multicolumn{6}{|l|}{ Lee et al. (35) } \\
\hline SMI loss vs. stable & & $0.16^{*}$ & & & $0.14^{*}$ \\
\hline \multicolumn{6}{|l|}{ SMI vs. SMI gain } \\
\hline SMRA loss vs. stable & 5-year survival $55.9 \%$ vs. $92.8 \%$ vs. $85.7 \%$ & $<0.001^{*}$ & 5-year surviva & vs. $94.0 \%$ vs. $90.5 \%$ & $p<0.001 *$ \\
\hline SMRA vs. SMRA gain & $2.32-29.23$ & $0.001 * *$ & 11.08 & $2.43-50.58$ & $0.002 * *$ \\
\hline
\end{tabular}

CT: Computed tomography; pts: patients; FIGO: International Federation of Gynecology and Obstetrics; SM: skeletal muscle; L3: third lumbar vertebra; SMA: lumbar psoas muscle cross-sectional area; SMI; skeletal muscle index; SMRA: skeletal muscle radiation attenuation; HU: Hounsfield Units; BMI: body mass index; NA: not available. 
Table VI. Baseline skeletal muscle assessment by CT scan in advanced ovarian carcinoma

\begin{tabular}{|c|c|c|c|c|c|}
\hline Authors & Pts & FIGO stage & Landmark & SM assessment & \\
\hline Aust et al. (39) & $140^{\mathrm{a}}$ & I-IV & L3 & $\begin{array}{l}\mathrm{SM} \leq 41 \mathrm{~cm}^{2} / \mathrm{m}^{2} \\
\text { SMRA }<39 \mathrm{HU}\end{array}$ & $\begin{array}{l}28.9 \% \\
35.0 \%\end{array}$ \\
\hline Kumar et al. (40) & $296^{\mathrm{a}}$ & IIIc-IV & L3 & $\begin{array}{c}\mathrm{SMI}<39 \mathrm{~cm}^{2} / \mathrm{m}^{2} \\
\text { Median SMRA }\end{array}$ & $\begin{array}{l}44.6 \% \\
33.4 \mathrm{HU}\end{array}$ \\
\hline Bronger et al. (41) & $105^{\mathrm{a}}$ & III-IV & L3 & $\mathrm{SMI}<38.5 \mathrm{~cm}^{2} / \mathrm{m}^{2}$ & $11 \%$ \\
\hline Rutten et al. (42) & $216^{\mathrm{a}}$ & IIb-IV & L3 & $\begin{array}{c}\text { SMI }<38.73 \mathrm{~cm}^{2} / \mathrm{m}^{2} \\
\text { Median SMRA }\end{array}$ & $\begin{array}{l}32.4 \% \\
36.64\end{array}$ \\
\hline Kim et al. (16) & $179^{\mathrm{b}}$ & III-IV & L3 & $\mathrm{SMI}<39 \mathrm{~cm}^{2} / \mathrm{m}^{2}$ & $42.5 \%$ \\
\hline Staley et al. (43) & $201^{b}$ & $\mathrm{I}-\mathrm{V}$ & L3 & $\mathrm{SMI}<41 \mathrm{~cm}^{2} / \mathrm{m}^{2}$ & $64.0 \%$ \\
\hline Atavesen et al. (44) & $323^{\mathrm{a}}$ & IIIb-IV & L3 & $\begin{array}{c}\mathrm{SMI}<38.5 \mathrm{~cm}^{2} / \mathrm{m}^{2} \\
\mathrm{SMI}<39 \mathrm{~cm}^{2} / \mathrm{m}^{2} \\
\mathrm{SMI}<41 \mathrm{~cm}^{2} / \mathrm{m}^{2} \\
\text { SMRA }<32 \mathrm{HU}\end{array}$ & $\begin{array}{l}29.4 \% \\
33.7 \% \\
47.1 \% \\
21.1 \%\end{array}$ \\
\hline Huang et al. (45) & $139^{\mathrm{a}}$ & III & L3 & $\begin{array}{c}\mathrm{SMI}<39.2 \mathrm{~cm}^{2} / \mathrm{m}^{2} \\
\text { SMRA }<35.5 \mathrm{HU}\end{array}$ & $\begin{array}{l}33.8 \% \\
33.1 \%\end{array}$ \\
\hline Rutten et al. (26) & $123^{\mathrm{c}}$ & IIB-IV & L3 & $\mathrm{SMI}<41.5 \mathrm{~cm}^{2} / \mathrm{m}^{2}$ & $50.4 \%$ \\
\hline Yoshino et al. (46) & $60^{c}$ & III-IV & L3 & $\begin{array}{c}\mathrm{SMI}<39 \mathrm{~cm}^{2} / \mathrm{m}^{2} \\
\text { SMRA }<0.96\end{array}$ & $\begin{array}{l}60 \% \\
43 \%\end{array}$ \\
\hline Conrad et al. (47) & $102^{\mathrm{a}}$ & III-IV & L4 & Mean CMI $2.8 \mathrm{~cm}^{2} / \mathrm{m}^{2}$ & \\
\hline
\end{tabular}

aPrimary debulking surgery; beither primary debulking surgery or interval debulking surgery; cinterval surgery. CT: Computed tomography; pts: patients; FIGO: International Federation of Gynecology and Obstetrics; SM: skeletal muscle; L3: third lumbar vertebra; SMI: skeletal muscle index; SMRA: skeletal muscle radiation attenuation; HU: Hounsfield Units; SMRA: pre- and post-neoadjuvant chemotherapy; SM area ratio; CMI: core muscle index.

$(\mathrm{HR}=2.957, p=0.006)$ and a trend to a lower OS $(\mathrm{HR}=2.572, p=0.06)$.

In another retrospective Japanese investigation, baseline skeletal muscle and psoas muscle were not associated with the clinical outcome (28). Conversely, the loss of skeletal muscle $\geq 15 \%$ after concurrent chemo-radiotherapy correlated with poorer PFS $(\mathrm{HR}=4.714, p=0.001)$ and poorer OS $(\mathrm{HR}=6.035$, $p=0.001)$ at univariate analysis, and the loss of psoas muscle $>15 \%$ was an independent poor prognostic factor for both PFS $(\mathrm{HR}=6.001, p=0.002)$ and $\mathrm{OS}(\mathrm{HR}=8.515, p=0.002)$.

\section{Endometrial Cancer}

Pretreatment sarcopenia has been reported in $33.6 \%-50 \%$ of patients with endometrial cancer (8,34-36) (Table IV). Conflicting data are currently available as for the prognostic relevance of SMI and SMRA (Table V).

Kuroki et al. (8) noted that $50 \%$ of surgically-treated patients had sarcopenia, defined as a CT-measured lumbar psoas muscle cross-sectional area $<4.33 \mathrm{~cm}^{2}$, and that $22 \%$ had sarcopenic obesity defined as sarcopenia plus BMI $>30 \mathrm{~kg} / \mathrm{m}^{2}$. There were no significant differences between sarcopenic and non-sarcopenic patients as for hospital stay, early and late complications and tumor features such as histological type, tumor grade, stage or microsatellite instability. Sarcopenia was an independent poor prognostic variable for PFS (HR=3.99) but not for OS.
A retrospective cohort study subdivided endometrial cancer patients according to whether SMI and SMRA were below or above the median values of $42.45 \mathrm{~cm}^{2} / \mathrm{m}^{2}$ and $30 \mathrm{HU}$, respectively (34). Longer median OS was observed among patients with high SMI and high SMRA as well as with low SMI and high SMRA, whereas shorter median OS was found among those with low SMI and low SMRA. At Cox regression analysis only the low SMI and low SMRA phenotype correlated with 1-year mortality $(\mathrm{HR}=5.31, p=0.004)$.

Lee et al. (35) assessed patients with stage III endometrial cancer who underwent total hysterectomy, bilateral salpingooophorectomy and lymphadenectomy followed by 3 cycles of paclitaxel $175 \mathrm{mg} / \mathrm{m}^{2}+$ carboplatin area under curve (AUC) 5 every 3 weeks, external beam radiotherapy plus brachytherapy, and 3 additional cycles of paclitaxel + carboplatin. In the entire cohort 5-year PFS was 76.1\% and 5-year OS was 79.7\%. Baseline SMI and SMRA did not correlate with PFS and OS. During treatment, patients lost an average of $2.1 \%$ of SMRA/210 days [ $95 \%$ confidence interval (CI): -4.0 to -0.2 ] and of $0.2 \%$ of SMI $/ 210$ days, but changes in SMRA and SMI did not correlate with changes in BMI ( $p=0.13$ and $p=0.20$, respectively). SMRA loss had a detrimental impact on both PFS $(\mathrm{HR}=8.24$, $p=0.001)$ and $\mathrm{OS}(\mathrm{HR}=11.08, p=0.002)$ at multivariate analysis, whereas SMI changes had no prognostic relevance.

Ganju et al. (36), who retrospectively assessed 64 patients treated with surgery and radiotherapy and with or 
Table VII. Prognostic relevance of skeletal muscle assessment by CT scan in advanced ovarian carcinoma. Baseline assessment.

\begin{tabular}{|c|c|c|c|c|c|c|}
\hline \multirow[t]{2}{*}{ Author } & \multicolumn{2}{|c|}{ Clinical outcome PFS } & \multirow[b]{2}{*}{$p$-Value } & \multicolumn{2}{|c|}{ Clinical outcome OS } & \multirow[b]{2}{*}{$p$-Value } \\
\hline & $\mathrm{HR}$ & $95 \% \mathrm{CI}$ & & $\mathrm{HR}$ & $95 \% \mathrm{CI}$ & \\
\hline \multicolumn{7}{|l|}{ Aust et al. (39) } \\
\hline \multirow[t]{2}{*}{ Low vs. high SMI } & 1.13 & $0.75-1.81$ & $0.605^{*}$ & 0.92 & $0.50-1.98$ & $0.786^{*}$ \\
\hline & 1.31 & $0.76-2.26$ & $0.366 * *$ & 1.23 & $0.61-2.48$ & $0.565 * *$ \\
\hline \multirow[t]{2}{*}{ Low vs. high SMRA } & 1.54 & $1.01-2.34$ & $0.046^{*}$ & 2.41 & $0.24-0.70$ & $0.001 *$ \\
\hline & 1.22 & $0.69-2.17$ & $0.500 * *$ & 2.25 & $1.09-4.65$ & $0.028 * *$ \\
\hline \multicolumn{7}{|l|}{ Kumar et al. (40) } \\
\hline \multirow[t]{2}{*}{ Low vs. high SMI } & & & & 0.98 & $0.78-1.23$ & $0.85^{*}$ \\
\hline & & & & 1.26 & $1.08-1.46$ & $0.002 *$ \\
\hline Low vs. high SMRA & & & & 1.23 & $1.05-1.43$ & $0.009 * *$ \\
\hline \multirow{3}{*}{$\begin{array}{l}\text { Broger et al. (41) } \\
\text { Low } v s . \text { high SMI }\end{array}$} & \multicolumn{2}{|c|}{15 vs. 22 months } & & \multicolumn{2}{|c|}{23 vs. 48 months } & \\
\hline & 2.64 & $1.24-5.64$ & $0.012 *$ & 3.17 & $1.29-7.80$ & $0.012 *$ \\
\hline & 2.52 & $1.10-5.81$ & $0.030 * *$ & 2.89 & $1.11-7.54$ & $0.031 * *$ \\
\hline \multicolumn{7}{|l|}{ Rutten et al. (42) } \\
\hline \multirow[t]{2}{*}{ Low vs. high SMI } & & & & 1.536 & $1.105-2.134$ & $0.011^{*}$ \\
\hline & & & & 1.362 & $0.968-1.916$ & $0.076 * *$ \\
\hline \multirow[t]{2}{*}{ Low $v s$. high SMRA } & & & & 1.417 & $1.011-1.984$ & $0.043 *$ \\
\hline & & & & & & $\mathrm{ns}^{* *}$ \\
\hline \multirow{3}{*}{$\begin{array}{l}\text { Kim et al. (16) } \\
\text { Low } v s . \text { high SMI }\end{array}$} & \multicolumn{2}{|c|}{ Median: 18.3 vs. 18.7 months } & \multicolumn{4}{|c|}{ 5-year survival 64.1 vs. 59.3\% } \\
\hline & 0.879 & $0.629-1.228$ & $0.451^{*}$ & 0.747 & $0.436-1.280$ & $0.289 *$ \\
\hline & 1.292 & $0.906-1.843$ & $0.157 * *$ & 0.870 & $0.488-1.550$ & $0.636^{* *}$ \\
\hline Among sarcopenic pts & \multicolumn{2}{|c|}{ Median: 18.3 vs. 18.2 months } & & 5-year sur & 7 vs. $80.0 \%$ & \\
\hline $\mathrm{FMR} \geq 2.1 \mathrm{vs.}<2.1$ & 1.262 & $0.762-2.092$ & $0.366^{*}$ & 2.476 & $0.989-6.199$ & $0.053 *$ \\
\hline & 1.073 & $0.576-1.999$ & $0.825 * *$ & 3.377 & $1.170-9.752$ & $0.024 * *$ \\
\hline Staley et al. (43) & Median: & .1 months, & $0.37 *$ & Median: 2 & 26.7 months & $0.8^{*}$ \\
\hline Low vs. high SMI & & & & & & \\
\hline Atavensen et al. (44) & & & & & & \\
\hline $\mathrm{SMI}<38.5 v s . \geq 38.5 \mathrm{~cm}^{2} / \mathrm{m}^{2}$ & & & & 471 & onths & $0.838 *$ \\
\hline$<39 v s . \geq 39 \mathrm{~cm}^{2} / \mathrm{m}^{2}$ & & & & 471 & onths & $0.613 *$ \\
\hline$<41$ vs. $\geq 41 \mathrm{~cm}^{2} / \mathrm{m}^{2}$ & & & & 501 & onths & $0.730 *$ \\
\hline SMRA low vs. high & & & & 281 & onths & $0.001 *$ \\
\hline & & & & 1.79 & $1.22-3.63$ & $0.003 * *$ \\
\hline Huang et al. (45) & & & & & & \\
\hline Low vs. high SMI & 5 -year I & vs. $38.5 \%$ & $0.03 *$ & 5-year OS & vs. $63.2 \%$ & $0.08 *$ \\
\hline & $1.03^{\circ}$ & $1.01-1.06$ & $0.04 * *$ & $1.08^{\circ}$ & $1.03-1.11$ & $0.002 * *$ \\
\hline Low $v s$. high SMRA & 5 -year I & vs. $37.7 \%$ & $0.24 *$ & 5-year Os & vs. $65.7 \%$ & $0.02 *$ \\
\hline & $1.04^{\circ \circ}$ & $1.01-1.09$ & $0.03 * *$ & $1.04^{\circ 0}$ & $0.99-1.10$ & $0.13 * *$ \\
\hline Loss $v s$. no SMI loss & 5 -year I & vs. $43.4 \%$ & $<0.001 *$ & 5-year Os & ovs. $68.8 \%$ & $0.001 *$ \\
\hline & $1.04^{\circ 00}$ & $1.01-1.06$ & $0.003 * *$ & $1.04^{\circ 00}$ & $1.01-1.08$ & $0.002 * *$ \\
\hline SMRA loss $v s$. no loss & 5 -year I & vs. $38.5 \%$ & $0.02 *$ & 5-year OS & vs. $67.2 \%$ & $0.10 *$ \\
\hline & $1.02^{\circ 00}$ & $0.99-1.04$ & $0.11 * *$ & & & \\
\hline Rutten et al. (26) & & & & & & \\
\hline Low vs. high SMI & & & & 0.887 & $0.556-1.414$ & $0.613 *$ \\
\hline SM loss during CT & & & & 2.218 & $1.280-3.844$ & $0.005 *$ \\
\hline & & & & 1.773 & $1.018-3.088$ & $0.043 * *$ \\
\hline Joshino et al. (46) & & & & & & \\
\hline Low vs. high SMI & & & & Not ass & with OS & $0.12 *$ \\
\hline Low $v s$. high SMAR & & & & & & $0.025 *$ \\
\hline & & & & 3.17 & $1.18-9.06$ & $0.022 * *$ \\
\hline
\end{tabular}


Table VII. Continued

\begin{tabular}{|c|c|c|c|c|c|c|}
\hline \multirow[t]{2}{*}{ Author } & \multicolumn{2}{|c|}{ Clinical outcome PFS } & \multirow[b]{2}{*}{$p$-Value } & \multicolumn{2}{|c|}{ Clinical outcome OS } & \multirow[b]{2}{*}{$p$-Value } \\
\hline & HR & $95 \% \mathrm{CI}$ & & HR & $95 \% \mathrm{CI}$ & \\
\hline \multicolumn{7}{|l|}{ Conrad et al. (47) } \\
\hline Low vs. high CMI & & & \multicolumn{4}{|c|}{ Similar OS } \\
\hline Low CMI+hypoalbunemia & & & \multicolumn{4}{|c|}{ Poorer OS } \\
\hline vs. Low CMI+normal albumin & & & & 3.75 & $1.15-9.38$ & $0.02 *$ \\
\hline
\end{tabular}

${ }^{\circ} 1 \mathrm{~cm}^{2} / \mathrm{m}^{2}$ decrease; ${ }^{\circ} 1 \mathrm{HU}$ decrease; ${ }^{\circ 00}$ per $1 \% / 180$ days decrease; *univariate analysis; $* *$ multivariate analysis. CT: Computed tomography; PFS: progression-free survival; SMI: skeletal muscle index; HR: hazard ratio; 95\%CI:95\% confidence interval: NA: not available; FMR, fat-to-muscle ratio: SM: skeletal muscle; SMRA: pre- and post-neoadjuvant chemotherapy SM area ratio; CMI: core muscle index.

without chemotherapy, reported that patients with low SMI and low SMRA were less likely to complete the planned chemotherapy $(p<0.01)$ whereas radiotherapy was well tolerated regardless of SMI or SRMA. Three-year OS was $29 \%$ for the patients with both low SMI and low SMRA, $75 \%$ for those with low SMI and $75 \%$ for those with low SMRA. The patients with low SMI and low SMRA experienced the worst $\mathrm{OS}(\mathrm{HR}=3.02, p=0.04)$ at multivariate analysis. In the study of Lee et al. (35) skeletal muscle gauge (SMG), i.e. the product of SMI per SMRA, was significantly associated with treatment delays, dose reductions, and discontinuation of chemotherapy, whereas the association of either SMI or SMRA with such changes was of borderline significance. Similarly, SMG was a better predictor of severe chemotherapy toxicity compared with either SMI or SMRA alone both in breast cancer women treated with an anthracycline/taxane-based regimen (37) and in colorectal cancer patients treated with 5-fluorouracil (38).

\section{Ovarian Cancer}

Baseline low SMI and low SMRA have been detected in 11\%$64 \%$ and $21.1 \%-35 \%$ of patients with advanced ovarian cancer, respectively (Table VI) $(16,26,39-47)$. The prognostic relevance of SMI and SMRA is reported in Table VII.

In an Austrian study, baseline SMI correlated with neither PFS nor OS (39). Conversely an elevated pretreatment SMRA was associated with a higher complete cytoreduction rate $(60.4 \%$ versus $42.9 \%, p=0.046)$, a longer PFS at univariate analysis $(\mathrm{HR}=1.54, p=0.046)$ and a longer $\mathrm{OS}$ at multivariate analysis $(\mathrm{HR}=2.25, p=0.028)$. The patients with low SMRA had poor nutritional status, decreased albumin levels, and systemic inflammatory status.

In a series of patients with advanced ovarian cancer who underwent primary debulking surgery at Mayo Clinic, median OS was 33.2 months with no difference between sarcopenic and non-sarcopenic patients (40).
Conversely, the risk of death increased linearly with decreasing values of SMRA, and at multivariate analysis the HR per $10 \mathrm{HU}$ decrease in SMRA was $1.23(p=0.009)$. Among patients without residual disease, median OS was significantly better for patients with SMRA $\geq 27.66 \mathrm{HU}$ compared with those with SMRA<27.66 HU. Similarly, among patients with residual disease median, OS was significantly longer for patients with SMRA $\geq 36.40 \mathrm{HU}$ compared with those with SMRA $<36.40$ HU. Therefore, SMRA related to lipid content and quality of skeletal muscle, seemed to have a greater prognostic relevance than skeletal muscle mass itself.

A German study found that baseline sarcopenia was an independent poor prognostic variable for PFS $(\mathrm{HR}=2.52$, $p=0.030)$ and $\mathrm{OS}(\mathrm{HR}=2.89, p=0.031)$, whereas skeletal muscle mass changes over time did not correlate with OS (41).

A Dutch study (42) reported that complete cytoreduction rates $(25.7 \%$ versus $35.6 \% p=0.346)$ and major surgical complication rates were not significantly different between sarcopenic and non-sarcopenic patients who underwent primary debulking surgery. Both baseline sarcopenia and low SMRA were predictors of poorer $\mathrm{OS}$ at univariate ( $\mathrm{HR}=1.536, p=0.011$ and $\mathrm{HR}=1.417, p=0.043$, respectively) but not at multivariate analysis.

A Korean study assessed patients who underwent primary debulking surgery or interval debulking surgery and platinum/taxane-based chemotherapy (16). Baseline SMI itself did not correlate with the clinical outcome. However, among sarcopenic patients, a high fat-to-muscle ratio (FMR) was an independent poor prognostic variable for OS $(\mathrm{HR}=3.377$, $p=0.024$ ) but not for PFS. Adipose stem cells from visceral and subcutaneous fat could enhance the proliferation and migration of ovarian cancer cells through the IL-6/STAT3 signaling pathway (48). Both sarcopenia and visceral obesity have been correlated with a chronic inflammatory state (49, $50)$. However, in the present Korean study SMI and SMRA were significantly associated with BMI ( $p<0.001$ for both), but not with neutrophil-to-lymphocyte ratio, monocyte-to- 
lymphocyte ratio, and platelet-to-lymphocyte ratio, which represent systemic inflammatory indices (16).

In the study of Staley et al. (43), median PFS and median OS were similar in sarcopenic and non-sarcopenic women at diagnosis. Moreover, no significant differences in chemotherapy toxicity, dose reduction and treatment delay according to SMI were detected among the 134 patients of whom chemotherapy records were available. Only a trend toward a more frequent neutropenia was noted in the sarcopenic group $(82.2 \%$ versus $65.6 \%, p=0.07)$.

Ataseven et al. (44) found that preoperative SMI with any cut off value was not associated with OS in a series of patients undergoing primary debulking surgery. Conversely, preoperative SMRA $<32$ HU correlated with a lower complete surgical cytoreduction rate $(38.2 \%$ versus $68.2 \%)$ and independently predicted a poorer $\mathrm{OS}(\mathrm{HR}=1.79, p=0.003)$. It is noteworthy that SMRA correlated with OS in the subset of patients with residual disease after surgery $(\mathrm{HR}=1.87,95 \% \mathrm{CI}=1.13-3.10$, $p=0.015)$ but not in those who underwent a complete cytoreduction $(\mathrm{HR}=1.66,95 \% \mathrm{CI}=0.82-3.38, p=0.161)$.

A Chinese study reported a mean SMI loss of 1.8\%/180 days and a mean SMRA loss of 1.7\%/180 days in patients who underwent primary debulking surgery followed by platinum-based chemotherapy (45). Baseline SMI $\left(1 \mathrm{~cm}^{2} / \mathrm{m}^{2}\right.$ decrease; $\mathrm{HR}=1.03, p=0.04)$, SMI changes $(1 \% / 180$ days decrease; HR=1.04, $p=0.003$ ) and baseline SMRA (1 HU decrease, $\mathrm{HR}=1.04, p=0.03)$ were independently associated with poorer PFS, and baseline SMI $(\mathrm{HR}=1.08, p=0.002)$ and SMI changes $(\mathrm{HR}=1.04, p=0.002)$ were also independent prognostic variables for OS.

Baseline sarcopenia was not related to OS in patients treated with neoadjuvant chemotherapy and interval debulking surgery (26). Conversely, median OS was significantly lower in patients with reduced skeletal muscle compared with those with stable or increased skeletal muscle during chemotherapy $(916 \pm 99$ versus $1431 \pm 470$ days, $\mathrm{HR}=2.218,95 \% \mathrm{CI}=1.280-3.844, p=0.005)$. Skeletal muscle loss during chemotherapy independently predicted OS $(\mathrm{HR}=1.773, p=0.043)$. Similarly, in another study, the skeletal muscle area measured at the third lumbar vertebra level decreased significantly after neoadjuvant chemotherapy ( $p=0.019)$ and a low post-to pre-neoadjuvant chemotherapy skeletal muscle area ratio (SMAR) was found to be an independent poor predictor of OS $(\mathrm{HR}=3.17$; $p=0.022$ ) (46). Conversely, baseline sarcopenia was not associated with OS.

The areas of bilateral psoas muscles at the fifth lumbar vertebra level were measured by CT scan in 76 patients with ovarian cancer who received carboplatin/paclitaxel-based chemotherapy (51). The patients with psoas muscle crosssectional area normalized to height [core muscle index (CMI)] $<583 \mathrm{~mm}^{2} / \mathrm{m}^{2}$ had a 3.93 -fold higher risk of developing grade $\geq 2$ peripheral neuropathy compared with those with CMI $>583 \mathrm{~mm}^{2} / \mathrm{m}^{2}$. Conversely, this variable did not correlate with neutropenia and thrombocytopenia.

Preoperative sarcopenia, defined as a CMI below the mean value of $2.8 \mathrm{~cm}^{2} / \mathrm{m}^{2}$, correlated with low serum albumin levels $(p=0.0002)$ but not with short-term morbidity and $\mathrm{OS}$ in patients undergoing primary debulking surgery (47). Whereas in patients without sarcopenia albumin status did not impact $\mathrm{OS}(\mathrm{HR}=1.11$, 95\% CI=0.37-3.29 $p=0.85)$. Patients who had both sarcopenia and hypoalbuminemia had a 3.75-fold higher risk of death compared to sarcopenic patients with normal albuminemia $(p=0.02)$.

A meta-analysis of 8 studies revealed that both baseline SMI and SMRA significantly correlated with OS $(\mathrm{HR}=1.11$, $95 \% \mathrm{CI}=1.03-1.20, p=0.007$ and, respectively, $\mathrm{HR}=1.14$, $95 \% \mathrm{CI}=1.08-1.20, p<0.001)(26,39-42,44,47,52,53) . \mathrm{A}$ more recent meta-analysis of 6 studies showed that normal SMRA was associated with a better 5-year OS compared with low SMRA (odds ratio=2.3, 95\%CI=1.6-3.4, $p<0.001$ ), whereas sarcopenia did not significantly impact the clinical outcome $(26,39-42,44,54)$. However, all the individual studies had an overall high risk of bias and, moreover, the lack of standardized cut-offs for these variables made the interpretation of the results very difficult.

\section{Conclusion}

A consensus has not yet been reached as for the optimal cutoff values for skeletal muscle assessment in cancer patients (8). Some authors $(23,24,36,39,43)$ defined sarcopenia as an SMI of $<41.0 \mathrm{~cm}^{2} / \mathrm{m}^{2}$ and low SMRA as an attenuation of $<41$ $\mathrm{HU}$ in patients with BMI of $<25.0 \mathrm{~kg} / \mathrm{m}^{2}$ or $<33 \mathrm{HU}$ in patients with a BMI of $\geq 25.0 \mathrm{~kg} / \mathrm{m} 2$, whereas others used the lower cutoff of $38.5 \mathrm{~cm}^{2} / \mathrm{m}^{2}(25,32,41,44)$ and $39 \mathrm{~cm}^{2} / \mathrm{m}^{2}(16,40,44$, 46) for SMI. The wide range in the prevalence of baseline sarcopenia in patients with gynecological cancer depends on both the different cut-off values and the heterogeneity of the patient populations in the different studies.

The available data showed that baseline sarcopenia is not associated with the clinical outcome of patients with locally advanced cervical cancer, whereas a relevant skeletal muscle loss during chemo-radiotherapy is a poor prognostic variable for PFS and OS $(23,28,32)$.

As for patients with surgically treated endometrial cancer, baseline sarcopenia and/or low SMRA correlates with unfavorable prognosis in some studies $(8,34)$, but not in others $(35,36)$. Decreased SMRA after surgery, chemotherapy and radiotherapy was independently associated with shorter PFS and OS in patients with stage III disease (35).

Among patients with advanced ovarian cancer treated with surgery and chemotherapy, baseline SMRA is usually related to a poorer clinical outcome $(39,40,42,44,45)$. However, baseline sarcopenia has an unfavorable prognostic impact in 
some studies $(41,42,45)$, but not in others $(16,26,39,40$, $43,44,46,47)$. Two studies reported that skeletal muscle loss during neoadjuvant chemotherapy is detrimental for OS $(26,46)$.

In conclusion, the scanty literature data seem to suggest that baseline SMI and SMRA have an uncertain prognostic relevance in gynecological cancers, whereas their changes during treatment usually correlate with PFS and OS. Although there is no commonly accepted therapeutic strategy to prevent cancer-related skeletal muscle damage, pharmacotherapy, physical activity and nutritional supplements have been used in attempting to preserve skeletal muscle mass and quality in cancer patients (55-60). Several agents, including cytokine inhibitors, steroids such as medroxyprogesterone acetate and testosterone, nonsteroidal anti-inflammatory drugs such as celecoxib, branched-chain amino acids, eicosapentaenoic acid, vitamin/minerals, carnitine, and antiserotoninergic drugs, have been tested with uncertain results $(55,58,60)$. Progressive resistance training could reduce the sarcopenic body changes through downregulation of different pro-inflammatory cytokines involved in skeletal muscle loss (56). The ongoing NCT0233092 randomized trial is evaluating the impact of nutrition intervention, home-based exercise, and antiinflammatory drugs in preventing or attenuating cachexia in advanced cancer patients [Multimodal Exercise/Nutrition/Antiinflammatory Treatment for Cachexia Trial (MENAC)]. Welldesigned multicenter clinical trials are strongly warranted to assess the effects of pharmacological agents and physical exercise in the management of skeletal muscle damage in patients with gynecological cancer and especially in those with locally advanced cervical cancer and with advanced ovarian cancer.

\section{Conflicts of Interest}

The Authors declare no conflicts of interest in relation to this study.

\section{Authors' Contributions}

Conceptualization, Writing - original draft: AG; Data curation, Formal analysis, Methodology, Writing-review \& editing: AG, SC.

\section{References}

1 Evans WJ, Morley JE, Argilés J, Bales C, Baracos V, Guttridge D, Jatoi A, Kalantar-Zadeh K, Lochs H, Mantovani G, Marks D, Mitch WE, Muscaritoli M, Najand A, Ponikowski P, Rossi Fanelli F, Schambelan M, Schols A, Schuster M, Thomas D, Wolfe R and Anker SD: Cachexia: a new definition. Clin Nutr 27: 793-799, 2008. PMID: 18718696. DOI: 10.1016/j.clnu.2008.06.013

2 Vagnildhaug OM, Blum D, Wilcock A, Fayers P, Strasser F, Baracos VE, Hjermstad MJ, Kaasa S, Laird B and Solheim TS: The applicability of a weight loss grading system in cancer cachexia: A longitudinal analysis. J Cachexia Sarcopenia Muscle 8: 789-797, 2017. PMID: 28627024. DOI: 10.1002/jcsm. 12220
3 VanderVeen BN, Fix DK and Carson JA: Disrupted skeletal muscle mitochondrial dynamics, mitophagy, and biogenesis during cancer cachexia: A role for inflammation. Oxid Med Cell Longev 2017: 3292087, 2017. PMID: 28785374. DOI: $10.1155 / 2017 / 3292087$

4 Chacon-Cabrera A, Mateu-Jimenez M, Langohr K, Fermoselle C, García-Arumí E, Andreu AL, Yelamos J and Barreiro E: Role of PARP activity in lung cancer-induced cachexia: Effects on muscle oxidative stress, proteolysis, anabolic markers, and phenotype. J Cell Physiol 232: 3744-3761, 2017. PMID: 28177129. DOI: $10.1002 /$ jcp.25851

5 Pin F, Barreto R, Kitase Y, Mitra S, Erne CE, Novinger LJ, Zimmers TA, Couch ME, Bonewald LF and Bonetto A: Growth of ovarian cancer xenografts causes loss of muscle and bone mass: a new model for the study of cancer cachexia. J Cachexia Sarcopenia Muscle 9: 685-700, 2018. PMID: 30009406. DOI: 10.1002/jcsm.12311

6 Prado CM, Baracos VE, McCargar LJ, Reiman T, Mourtzakis M, Tonkin K, Mackey JR, Koski S, Pituskin E and Sawyer MB: Sarcopenia as a determinant of chemotherapy toxicity and time to tumor progression in metastatic breast cancer patients receiving capecitabine treatment. Clin Cancer Res 15: 2920-2926, 2009. PMID: 19351764. DOI: 10.1158/1078-0432.CCR-08-2242

7 Muscaritoli M, Anker SD, Argilés J, Aversa Z, Bauer JM, Biolo G, Boirie Y, Bosaeus I, Cederholm T, Costelli P, Fearon KC, Laviano A, Maggio M, Rossi Fanelli F, Schneider SM, Schols A and Sieber CC: Consensus definition of sarcopenia, cachexia and pre-cachexia: joint document elaborated by Special Interest Groups (SIG) "cachexia-anorexia in chronic wasting diseases" and "nutrition in geriatrics". Clin Nutr 29: 154-159, 2010. PMID: 20060626. DOI: 10.1016/j.clnu.2009.12.004

8 Kuroki LM, Mangano M, Allsworth JE, Menias CO, Massad LS, Powell MA, Mutch DG and Thaker PH: Pre-operative assessment of muscle mass to predict surgical complications and prognosis in patients with endometrial cancer. Surg Oncol 22: 972-979, 2015. PMID: 25190123. DOI: 10.1245/s10434-014-4040-8

9 Shachar SS, Deal AM, Weinberg M, Nyrop KA, Williams GR, Nishijima TF, Benbow JM and Muss HB: Skeletal muscle measures as predictors of toxicity, hospitalization, and survival in patients with metastatic breast cancer receiving taxane-based chemotherapy. Clin Cancer Res 23: 658-665, 2017. PMID: 27489287. DOI: $10.1158 / 1078-0432$.CCR-16-0940

10 Chan MY and ChokK SH: Sarcopenia in pancreatic cancer effects on surgical outcomes and chemotherapy. World J Gastrointest Oncol 11: 527-537, 2019. PMID: 31367272. DOI: 10.4251/wjgo.v11.i7.527

11 Matsuoka H, Nakamura K, Matsubara Y, Ida N, Nishida T, Ogawa C, Katsi K, Kanazawa S and Masuyama H: Sarcopenia is not a prognostic factor of outcome in patients with cervical cancer undergoing concurrent chemoradiotherapy or radiotherapy. Anticancer Res 39: 933-939, 2019. PMID: 30711978. DOI: 10.21873 /anticanres.13196

12 Cruz-Jentoft AJ, Bahat G, Bauer J, Boirie Y, Bruyère O, Cederholm T, Cooper C, Landi F, Rolland Y, Sayer AA, Schneider SM, Sieber CC, Topinkova E, Vandewoude M, Visser $\mathrm{M}$ and Zamboni M: Writing Group for the European Working Group on Sarcopenia in Older People 2 (EWGSOP2), and the Extended Group for EWGSOP2. Sarcopenia: revised European consensus on definition and diagnosis. Age Ageing 48: 16-31, 2019. PMID: 30312372. DOI: 10.1093/ageing/afy169 
13 Aro R, Mäkäräinen-Uhlbäck E, Ämmälä N, Rautio T, Ohtonen $\mathrm{P}$, Saarnio $\mathrm{J}$ and Meriläinen S: The impact of sarcopenia and myosteatosis on postoperative outcomes and 5-year survival in curatively operated colorectal cancer patients- A retrospective register study. Eur J Surg Oncol 46: 1656-1662, 2020. PMID: 32224072. DOI: 10.1016/j.ejso.2020.03.206

14 Deng L, Wang Y, Zhao J, Tong Y, Zhang S, Jin C, Chen K, Bao W, Yu Z and Chen G: The prognostic value of sarcopenia combined with hepatolithiasis in intrahepatic cholangiocarcinoma patients after surgery: A prospective cohort study. Eur J Surg Oncol S0748-7983(20): 30724-1, 2020. PMID: 32933804. DOI: $10.1016 /$ j.ejso.2020.09.002

15 Chargi N, Bril SI, Swartz JE, Wegner I, Willems SM and de Bree R: Skeletal muscle mass is an imaging biomarker for decreased survival in patients with oropharyngeal squamous cell carcinoma. Oral Oncol 101: 104519, 2020. PMID: 31855705. DOI: $10.1016 /$ j.oraloncology.2019.104519

16 Kim SI, Kim TM, Lee M, Kim HS, Chung HH, Cho JY and Song YS: Impact of CT-Determined sarcopenia and body composition on survival outcome in patients with advancedstage high-grade serous ovarian carcinoma. Cancers (Basel) 12: 559, 2020. PMID: 32121099 . DOI: 10.3390/cancers 12030559

17 Allanson ER, Peng Y, Choi A, Hayes S, Janda M and Obermair A: A systematic review and meta-analysis of sarcopenia as a prognostic factor in gynecological malignancy. Int J Gynecol Cancer 30: 1791-1797, 2020. PMID: 32747410. DOI: 10.1136/ ijgc-2020-001678

18 Chen LK, Woo J, Assantachai P, Auyeung TW, Chou MY, Iijima K, Jang HC, Kang L, Kim M, Kim S, Kojima T, Kuzuya M, Lee JSW, Lee SY, Lee WJ, Lee Y, Liang CK, Lim JY, Lim WS, Peng LN, Sugimoto K, Tanaka T, Won CW, Yamada M, Zhang T, Akishita $\mathrm{M}$ and Arai H: Asian working group for sarcopenia: 2019 consensus update on sarcopenia diagnosis and treatment. J Am Med Dir Assoc 21: 300-307, 2020. PMID: 32033882. DOI: 10.1016/j.jamda.2019.12.012

19 Rutten IJG, Ubachs J, Kruitwagen R, Beets-Tan RGH, OldeDamink SWM and Van Gorp T: Psoas muscle area is not representative of total skeletal muscle area in the assessment of sarcopenia in ovarian cancer. J. Cachexia Sarcopenia Muscle 8: 630-638, 2017. PMID: 28513088. DOI: $10.1002 /$ jcsm. 12180

20 Mitsiopoulos N, Baumgartner RN, Heymsfield SB, Lyons W, Gallagher D and Ross R: Cadaver validation of skeletal muscle measurement by magnetic resonance imaging and computerized tomography. J Appl Physiol 85: 115-122, 1998. PMID: 9655763. DOI: 10.1152 jappl.1998.85.1.115

21 Mourtzakis M, Prado CM, Lieffers JR, Reiman T, McCargar LJ and Baracos VE: A practical and precise approach to quantification of body composition in cancer patients using computed tomography images acquired during routine care. Appl Physiol Nutr Metab 33: 997-1006, 2008. PMID: 18923576. DOI: 10.1139/H08-075

22 Shen W, Punyanitya M, Wang Z, Gallagher D, St-Onge MP, Albu J, Heymsfield SB, and Heshka S: Total body skeletal muscle and adipose tissue volumes: estimation from a single abdominal cross-sectional image. J Appl Physiol 97: 2333-2338, 2004. PMID: 15310748. DOI: 10.1152/japplphysiol.00744.2004

23 Lee J, Chang CL, Lin JB, Wu MH, Sun FJ, Jan YT, Hsu SM and Chen YJ: Skeletal muscle loss is an imaging biomarker of outcome after definitive chemoradiotherapy for locally advanced cervical cancer. Clin Cancer Res 24: 5028-5036, 2018. PMID: 29959140. DOI: 10.1158/1078-0432.CCR-18-0788

24 Martin L, Birdsell L, Macdonald N, Reiman T, Clandinin MT, McCargar LJ, Murphy R, Ghosh S, Sawyer MB and Baracos VE: Cancer cachexia in the age of obesity: skeletal muscle depletion is a powerful prognostic factor, independent of body mass index. J Clin Oncol 31: 1539-1547, 2013. PMID: 23530101. DOI: $10.1200 / \mathrm{JCO} .2012 .45 .2722$

25 Prado CM, Lieffers JR, McCargar LJ, Reiman T, Sawyer MB, Martin L and Baracos VE: Prevalence and clinical implications of sarcopenic obesity in patients with solid tumours of the respiratory and gastrointestinal tracts: a population-based study. Lancet Oncol 9: 629-635, 2008. PMID: 18539529. DOI: 10.1016/S1470-2045(08)70153-0

26 Rutten IJ, van Dijk DP, Kruitwagen RF, Beets-Tan RG, Olde Damink SW and van Gorp T: Loss of skeletal muscle during neoadjuvant chemotherapy is related to decreased survival in ovarian cancer patients. J. Cachexia Sarcopenia Muscle 7: 458466, 2016. PMID: 27030813. DOI: 10.1002/jcsm.12107

27 Daly LE, Ni Bhuachalla EB, Power DG, Cushen SJ, James K and Ryan AM: Loss of skeletal muscle during systemic chemotherapy is prognostic of poor survival in patients with foregut cancer. J Cachexia Sarcopenia Muscle 9: 315-325, 2018. PMID: 29318756. DOI: 10.1002/jcsm.12267

28 Kiyotoki T, Nakamura K, Haraga J, Omichi C, Ida N, Saijo M, Nishida T, Kusumoto $\mathrm{T}$ and Masuyama H: Sarcopenia is an important prognostic factor in patients with cervical cancer undergoing concurrent chemoradiotherapy. Int J Gynecol Cancer 28: 168-175, 2018. PMID: 29040185. DOI: 10.1097/IGC.0000 000000001127

29 Aubrey J, Esfandiari N, BaracosVE, Buteau FA, Frenette J, Putman CT and Mazurak VC: Measurement of skeletal muscle radiation attenuation and basis of its biological variation. Acta Physiol 210: 489-497, 2014. PMID: 24393306. DOI: 10.1111/ apha.12224

30 Stephens NA, Skipworth RJ, MacDonald AJ, Greig CA, Ross JA and Fearon KC: Intramyocellular lipid droplets increase with progression of cachexia in cancer patients. J Cachexia Sarcopenia Muscle 2: 111-117, 2011. PMID: 21766057. DOI: $10.1007 / \mathrm{s} 13539-011-0030-\mathrm{x}$

31 Goodpaster BH, Kelley DE, Thaete FL, He J and Ross R: Skeletal muscle attenuation determined by computed tomography is associated with skeletal muscle lipid content. J Appl Phys 89: 104110, 2000. PMID: 10904041. DOI: 10.1152/jappl.2000.89.1.104

32 Sánchez M, Castro-Eguiluz D, Luvián-Morales J, Jiménez-Lima R, Aguilar-Ponce JL, DIsla-Ortiz D and Cetina L: Deterioration of nutritional status of patients with locally advanced cervical cancer during treatment with concomitant chemoradiotherapy. J Hum Nutr Diet 32: 480-491, 2019. PMID: 30938007. DOI: 10.1111/jhn.12649

33 Gadducci A, Guerrieri ME and Cosio S: Adenocarcinoma of the uterine cervix: pathologic features, treatment options, clinical outcome and prognostic variables. Crit Rev Oncol Hematol 135: 103-114, 2019. PMID: 30819439. DOI: 10.1016/j.critrevonc. 2019.01.006

34 Rodrigues CS and Chaves GV: Skeletal muscle quality beyond average muscle attenuation: aproposal of skeletal muscle phenotypes to predict short-term survival in patients with endometrial cancer. J Natl Compr Canc Netw 16: 153-160, 2018. PMID: 29439176. DOI: 10.6004/jncen.2017.7028 
35 Lee J, Lin JB, Wu MH, Jan YT, Chang CL, Huang CY, Sun FJ and Chen YJ: Muscle radiodensity loss during cancer therapy is predictive for poor survival in advanced endometrial cancer. J Cachexia Sarcopenia Muscle 10: 814-826, 2019. PMID: 31094101. DOI: $10.1002 /$ jcsm. 12440

36 Ganju RG, TenNapel M, Spoozak L, Chen AM and Hoover A: The impact of skeletal muscle abnormalities on tolerance to adjuvant chemotherapy and radiation and outcome in patients with endometrial cancer. J Med Imaging Radiat Oncol 64: 104112, 2020. PMID: 31397078. DOI: 10.1111/1754-9485.12935

37 Shachar SS, Deal AM, Weinberg M, Williams GR, Nyrop KA, Popuri K, Choi SK and Muss HB: Body composition as a predictor of toxicity in patients receiving anthracycline and taxane-based chemotherapy for early-stage breast cancer. Clin Cancer Res 23: 3537-3543, 2017. PMID: 28143874. DOI: 10.1158/1078-0432.CCR-16-2266

38 Williams GR, Deal AM, Shachar SS, Walko CM, Patel JN, O'Neil BH, McLeod HL, Weinberg MS, Choi SK, Muss HB and Sanoff HK: The impact of sarcopenia on toxicity and pharmacokinetics of 5-fluorouracil (5FU) in colorectal cancer. Cancer Chemother Pharmacol 81: 413-417, 2018. PMID: 29159476. DOI: 10.1007/s00280-017-3487-2

39 Aust S, Knogler T, Pils D, Obermayr E, Reinthaller A, Zahn L, Radlgruber I, Mayerhoefer ME, Grimm C and Polterauer S: Skeletal muscle depletion and markers for cancer cachexia are strong prognostic factors in epithelial ovarian cancer. PLoS One 10: e0140403, 2015. PMID: 26457674. DOI: 10.1371/journal. pone. 0140403

40 Kumar A, Moynagh MR, Multinu F, Cliby WA, McGree ME, Weaver AL, Young PM, Bakkum-Gamez JN, Langstraat CL, Dowdy SC, Jatoi A and Mariani A: Muscle composition measured by CT scan is a measurable predictor of overall survival in advanced ovarian cancer. Gynecol Oncol 142: 311316, 2016. PMID: 27235857. DOI: 10.1016/j.ygyno.2016.05.027

41 Bronger H, Hederich P, Hapfelmeier A, Metz S, Noël PB, Kiechle M and Schmalfeldt B: Sarcopenia in advanced serous ovarian cancer. Int J Gynecol Cancer 27: 223-232, 2017. PMID: 27870708. DOI: $10.1097 /$ IGC.0000000000000867

42 Rutten IJ, Ubachs J, Kruitwagen RF, van Dijk DP, Beets-Tan RG, Massuger LF, OldeDamink SW and Van Gorp T: The influence of sarcopenia on survival and surgical complications in ovarian cancer patients undergoing primary debulking surgery. Eur J Surg Oncol 43: 717-724, 2017. PMID: 28159443. DOI: 10.1016/j.ejso.2016.12.016

43 Staley SA, Tucker K, Newton M, Ertel M, Oldan J, Doherty I, West L, Zhang Y and Gehrig PA: Sarcopenia as a predictor of survival and chemotoxicity in patients with epithelial ovarian cancer receiving platinum and taxane-based chemotherapy. Gynecol Oncol 156: 695-700, 2020. PMID: 31928805. DOI: 10.1016/j.ygyno.2020.01.003

44 Ataseven B, Luengo TG, du Bois A, Waltering KU, Traut A, Heitz F, Alesina PF, Prader S, Meier B, Schneider S, Koch JA, Walz M, Groeben HT, Nina P, Brunkhorst V, Heikaus S and Harter P: Skeletal muscle attenuation (sarcopenia) predicts reduced overall survival in patients with advanced epithelial ovarian cancer undergoing primary debulking surgery. Ann Surg Oncol 25: 3372-3379, 2018. PMID: 30069659. DOI: 10.1245/ s10434-018-6683-3

45 Huang CY, Yang YC, Chen TC, Chen JR, Chen YJ, Wu MH, Jan YT, Chang CL and Lee J: Muscle loss during primary debulking surgery and chemotherapy predicts poor survival in advancedstage ovarian cancer. J Cachexia Sarcopenia Muscle 11: 534546, 2020. PMID: 31999069. DOI: 10.1002/jcsm.12524

46 Yoshino Y, Taguchi A, Nakajima Y, Takao M, Kashiyama T, Furusawa A, Kino N and Yasugi T: Extreme skeletal muscle loss during induction chemotherapy is an independent predictor of poor survival in advanced epithelial ovarian cancer patients. J Obstet Gynaecol Res 46: 2662-2671, 2020. PMID: 33015913. DOI: $10.1111 /$ jog. 14516

47 Conrad LB, Awdeh H, Acosta-Torres S, Conrad SA, Bailey AA, Miller DS and Lea JS: Pre-operative core muscle index in combination with hypoalbuminemia is associated with poor prognosis in advanced ovarian cancer. J Surg Oncol 117: 10201028, 2018. PMID: 29409111. DOI: 10.1002/jso.24990

48 Kim B, Kim HS, Kim S, Haegeman G, Tsang BK, Dhanasekaran DN and Song YS: Adipose stromal cells from visceral and subcutaneous fat facilitate migration of ovarian cancer cells via IL-6/JAK2/STAT3 pathway. Cancer Res Treat 49: 338-349, 2017. PMID: 27456942. DOI: $10.4143 /$ crt.2016.175

49 Wisse BE: The inflammatory syndrome: the role of adipose tissue cytokines in metabolic disorders linked to obesity. J Am Soc Nephrol 15: 2792-2800, 2004. PMID: 15504932. DOI: 10.1097/01.ASN.0000141966.69934.21

50 Dalle S, Rossmeislova L and Koppo K: The role of inflammation in age-related sarcopenia. Front Physiol 8: 1045, 2017. PMID: 29311975. DOI: 10.3389/fphys.2017.01045

51 Yoshikawa T, Takano M, Miyamoto M, Yajima I, Shimizu Y, Aizawa Y, Suguchi Y, Moriiwa M, Aoyama T, Soyama H, Goto T, Hirata J, Suzuki A, Sasa H, Nagaoka I, Tsuda H and Furuya $\mathrm{K}$ : Psoas muscle volume as a predictor of peripheral neurotoxicity induced by primary chemotherapy in ovarian cancers. Cancer Chemother Pharmacol 80: 555-561, 2017. PMID: 28726081. DOI: 10.1007/s00280-017-3395-5

52 de Paula NS, de Aguiar Bruno K, Aredes MA and Chaves GV: Sarcopenia and skeletal muscle quality as predictors of postoperative complication and early mortality in gynecologic cancer. Int J Gynecol Cancer 28: 412-420, 2018. PMID: 29266018. DOI: $10.1097 /$ IGC.0000000000001157

53 Ubachs J, Ziemons J, Minis-Rutten IJG, Kruitwagen RFPM, Kleijnen J, Lambrechts S, Olde-Damink SWM, Rensen SS and Van Gorp T: Sarcopenia and ovarian cancer survival: a systematic review and meta-analysis. J Cachexia Sarcopenia Muscle 10: 1165-1174, 2019. PMID: 31389674. DOI: 10.1002/ jcsm.12468.

54 McSharry V, Mullee A, McCann L, Rogers AC, McKiernan M and Brennan DJ: The impact of sarcopenia and low muscle attenuation on overall survival in epithelial ovarian cancer: a systematic review and meta-analysis. Ann Surg Oncol 27: 35533564, 2020. PMID: 32221737. DOI: 10.1245/s10434-02008382-0.

55 Suzuki H, Asakawa A, Amitani H, Nakamura N and Inui A: Cancer cachexia - pathophysiology and management J Gastroenterol 48: 574-594, 2013. PMID: 23512346. DOI: 10.1007/s00535-013-0787-0

56 Capozzi LC, McNeely ML, Lau HY, Reimer RA, Giese -Davis J, Fung TS and Culos-Reed SN: Patient-reported outcomes, body composition, and nutrition status in patients with head and neck cancer: Results from anexploratory randomized controlled exercise trial. Cancer 122: 1185-1200, 2016. PMID: 26828426. DOI: $10.1002 /$ cncr.29863 
57 Peterson SJ and Mozer M: Differentiating sarcopenia and cachexia among patients with cancer. Nutr Clin Pract 32: 30-39, 2017. PMID: 28124947. DOI: 10.1177/0884533616680354

58 Wright TJ, Dillon EL, Durham WJ, Chamberlain A, Randolph KM, Danesi C, Horstman AM, Gilkison CR, Willis M, Richardson G, Hatch SS, Jupiter DC, McCammon S, Urban RJ and Sheffield-Moore $\mathrm{M}$ : A randomized trial of adjunct testosterone for cancer-related muscle loss in men and women. J Cachexia Sarcopenia Muscle 9: 482-496, 2018. PMID: 2965464. DOI: $10.1002 /$ jcsm.12295

59 Dieli-Conwright CM, Courneya KS, Demark-Wahnefried W, Sami N, Lee K, Buchanan TA, Spicer DV, Tripathy D, Bernstein $\mathrm{L}$ and Mortimer JE: Effects of aerobic and resistance exercise on metabolic syndrome, sarcopenic obesity, and circulating biomarkers in overweight or obese survivors of breast cancer: a randomized controlled trial. J Clin Oncol 36: 875-883, 2018. PMID: 29356607. DOI: 10.1200/JCO.2017.75.7526

60 Prado CM, Purcell SA and Laviano A: Nutrition interventions to treat low muscle mass in cancer. J Cachexia Sarcopenia Muscle 11: 366-380, 2020. PMID: 31916411. DOI: 10.1002/jcsm.12525

Received December 7, 2020

Revised December 20, 2020

Accepted December 21, 2020 